EDITORIAL

\section{Grenzen der Therapie}

\author{
Hans-Ludwig Kröber
}

Online publiziert: 3. Juli 2013

(C) Springer-Verlag Berlin Heidelberg 2013

Strafe muss sein. Man kann zwar kunstvoll dagegen argumentieren, in friedlichen Hörsälen fern jeder Gewaltdrohung. Aber bereits dann, wenn man sich mit der Polizei in soziale Brennpunkte begibt, lernt man schnell, dass alles Deeskalieren, Versöhnen, Beruhigen, Beschwichtigen seine Grenzen hat, dass es bewaffnete Macht geben muss zur Durchsetzung der elementaren sozialen Regeln: körperliche Unversehrtheit, individuelle Freiheit, individueller Besitz. Bereits der Anschein, solches ließe sich nicht mehr durchsetzen, wäre fatal; zum Schutze der Rechtsordnung bedarf es der Strafe und des Strafvollzugs, vergeltend zum Schutz der Normgeltung und ebenso präventiv.

Im Sinne der „klassischen“ Strafrechtsschule war die Normgeltung mit dem Vollzug der schuldangemessenen Strafe wieder hergestellt; dem Strafvollzug kamen allenfalls aus allgemein-humanistischen oder religiösen Gründen Aufgaben dergestalt zu, dass der Täter zur Besinnung, Reue und Läuterung kommen möge. So wurde ihnen in manchen Strafanstalten ein Schweigegebot auferlegt und Kontakt zu Mitgefangenen systematisch unterbunden, damit sie sich auf sich selbst besinnen. Nach Strafverbüßung war der Verurteilte ein freier Mann und konnte frei entscheiden, hinfort nicht mehr zu sündigen.

Den Vätern der „modernen“ Strafrechtslehre wie Franz von Liszt verdanken wir den Gedanken, dass die Strafe v. a. präventiv wirken möge, was wiederum bedeutet, dass man auch den Strafvollzug nutzen könnte, um nicht nur durch Zufügung eines Übels - des kargen Lebens im Knast -, sondern durch einen Behandlungsvollzug auf ein verantwort-

H.-L. Kröber $(\bowtie)$

Institut für Forensische Psychiatrie,

Charité - Universitätsmedizin Berlin,

Oranienburger Str. 285, 13437 Berlin, Deutschland

E-Mail: Hans-Ludwig.Kroeber@charite.de liches Leben in Freiheit vorzubereiten. Dieser Gedanke ist frisch und lebendig seit 100 Jahren in der Diskussion über die Durchführung des Strafvollzugs; er hat einiges bewirkt in der Humanisierung des Strafvollzugs, weil man erkannt hat, dass rohe und entwürdigende Behandlung und Unterbringung rohe und entwürdigte Gefangene schafft.

Man hat auch schon früh über weitergehende fördernde Maßnahmen nachgedacht, eher im pädagogischen Bereich: Beschulung, Berufsausbildung, Erwerb von Fertigkeiten, aber auch so etwas wie ein soziales Kompetenztraining. Psychisch kranke Rechtsbrecher, das war klar, sollten in psychiatrischen Kliniken behandelt werden; aber man wollte nun auch - so die Überlegungen in den 70er Jahren des 20. Jahrhunderts - die sozial Schwachen, die Dissozialen, die geprügelten und Heimkinder, die zu Straftätern geworden waren, sozialtherapeutisch fördern und ihnen helfen, ihre Sozialisationsdefizite und -narben zu überwinden. Dafür wurden die sozialtherapeutischen Anstalten eingerichtet, nach längeren Diskussionen nicht als eigenständige, gerichtlich anzuordnende Maßregel wie Psychiatrie oder Suchtklinik, sondern als eine spezielle Option im Rahmen des Strafvollzugs.

Inzwischen sind die oft rückfälligen dissozialen Eigentums- und Gewaltdelinquenten aus den sozialtherapeutischen Abteilungen weitgehend verdrängt zugunsten der Sexualstraftäter, die ein Anrecht auf Sozialtherapie besitzen, sowie von Tätern mit schweren Gewalttaten.

Und vor unseren Augen vollzieht sich in diesen Tagen, dass gemäß den Wünschen des Bundesverfassungsgerichts der Gesetzgeber eine Sozialtherapie $2.0 \mathrm{schafft}$, nämlich neue Bauten, neues Personal und ein neues Behandlungssetting für Sicherungsverwahrte, die alle unter Erfolgszwang zu therapieren sind: Ist die Therapie nicht geeignet, diesen Verwahrten zum Erfolg zu führen, ist er auch bei fortgesetzter Gefährlichkeit in Freiheit zu entlassen. Existiert allerdings 
keine geeignete Therapie, bleibt er. Niedersachsen baut 37 Plätze für EUR 12,5 Mio. in Rosdorf, in Bützow/Mecklenburg gibt es 20 Plätze für EUR 7 Mio., in Bautzen wurden bislang EUR 7,4 Mio. verbaut, in Straubing wurden EUR 26 Mio. für einen Neubau investiert. Nahezu jedes Bundesland baut neu, weil die vorhandenen Zellen zu klein sind. Pro Verwahrten werden wohl etwa EUR 200-300.000 investiert.

Neue, bessere Gefängnisbauten sind keine schlechte Sache; die kann man immer gebrauchen. Das Problem ist: Wie will man sie mit Therapeuten füllen? Und was sollen sie tun mit den Sicherungsverwahrten? Das Problem beginnt damit, dass die Therapie der zur Sicherungsverwahrung Verurteilten ja bereits in der Strafhaft, möglichst früh beginnen soll. Also lange bevor sie die neuen Häuser beziehen. Wenn sie die neuen Häuser beziehen, ist offenbar die vorangehende Therapie gescheitert, jedenfalls erfolglos geblieben, und was macht man nun?

Das sehr nachdrückliche und optimistische Betonen des Therapieangebots für die Sicherungsverwahrten durch das Verfassungsgericht wie durch den Gesetzgeber wirft den Gedanken auf, ob hier in Wahrheit mit einer diabolischen List die Untauglichkeit von Straftätertherapie in einem Großversuch jedermann vor Augen geführt werden soll. Tatsächlich weiß ja keiner, wie man Sicherungsverwahrte wirksam, wirksamer als bisher, behandelt. Der Frage, ob die Therapie von Rückfalltätern eine große Illusion ist, widmete sich am 14.06.2013 die 17. Berliner Junitagung für Forensische Psychiatrie und Psychologie; es ging darum, Möglichkeiten, aber auch Grenzen auszuloten. Einige rechtzeitig vorliegende Beiträge der Junitagung sind in diesem Heft versammelt.

So beleuchtet der nunmehr Vorsitzende Richter am Bundesgerichtshof Thomas Fischer aus seiner Sicht die Etablierung des Therapiegedankens und die Brechungen im überlappenden Bereich von Strafe und Maßregel. Jörg Schäfer, Direktor der großen Justizvollzugsanstalt Diez, in der auch die Sicherungsverwahrten des Landes RheinlandPfalz in einem Neubau mit 43 Plätzen untergebracht sind, beschreibt sehr anschaulich die Probleme, die entstehen, wenn sich „Strafer“ zu „SVern“ wandeln, und wie im tatsächlichen Haftleben die von uns so stark fokussierten therapeutischen Angebote und Möglichkeiten vom Verurteilten eher beiläufig wahrgenommen werden. So wird eine wichtige Rolle spielen, welchen neuen Status sich Sicherungsver- wahrte in den nächsten Jahren über immer neue rechtliche Abklärungen ihrer Rechte und Pflichten verschaffen werden, und ob das Therapieangebot jemals den Stellenwert der Unterhaltungselektronik erreichen wird. In Berlin betreiben wir seit Monaten eine vollständige Erfassung wesentlicher Daten aller zu Sicherungsverwahrung Verurteilten; in diesem Heft diskutieren wir einige dieser Kenndaten wie Alter, Delikte, Haftdauer, vorangehende Therapieerfahrungen im Hinblick auf den tatsächlichen Interventionsbedarf und die möglichen Interventionsformen. Gestützt auf langjährige Therapieerfahrung in der Sozialtherapeutischen Anstalt Kassel II berichtet Thomas Thalmann über einige typische Therapiehemmnisse bei Straftätern und macht damit zugleich Mut, dass es durchaus eine Gruppe von Verurteilten gibt, bei denen gruppen- und auch einzeltherapeutisch wirkungsvoll gearbeitet werden kann. Die Hamburger Arbeitsgruppe um Frau Yoon und Peer Briken befasst sich mit den ambulanten Therapiemöglichkeiten für Sexualstraftäter; ,ambulant“ scheint weit weg zu sein von ,sicherungsverwahrt“, aber faktisch können wir manchen Verwahrten wahrscheinlich ambulant wesentlich wirksamer behandeln als unter Verschluss. Dem Thema fügt sich gut der Beitrag von Aglaja Stöver et al. an, weil der von ihnen dargestellte Jugendmaßregelvollzug an der Auseinandersetzung teilnimmt, was therapeutisch wirkt: ob jugendliche Straftäter einfach nur als soziale Abweichler sozialpädagogisch, als bildungsfern erzieherisch und enkulturierend oder als psychisch gestört jugendpsychiatrisch behandelt werden sollen.

Als freier Beitrag folgt die Fortsetzung des kriminologischen Beitrags von Michael Brockmann und Michael Bock zu Methodik und Praxis der Kriminalprognose, konkretisiert in Bezug auf einzelne Persönlichkeitsstörungen. Abgerundet wird dies mit einem ausführlichen Tagungsbericht über biologische Forschung in der forensischen Psychiatrie sowie unsere regelmäßigen Rubriken. Insgesamt soll das Heft nicht bange machen vor den Aufgaben, welche die Umgestaltung der Sicherungsverwahrung, aber letztlich auch des Strafvollzugs stellen werden. Aber es spricht alles dafür, genau hinzusehen, welche Lage sich darstellt, sich neue Gedanken über neue Probleme zu machen, neue Wege des Umgangs mit alten Rechtsbrechern zu finden und nicht schematisch zu handeln.

Hans-Ludwig Kröber 